\title{
Double-Differential Production Cross Sections of Charged Pions in Charged Pion Induced Nuclear Reactions at High Momentums
}

\author{
Ya-Qin Gao, ${ }^{1}$ Tian Tian, ${ }^{1}$ S. Fakhraddin, ${ }^{2,3}$ Magda A. Rahim,, ${ }^{2,3}$ and Fu-Hu Liu ${ }^{1}$ \\ ${ }^{1}$ Institute of Theoretical Physics, Shanxi University, Taiyuan, Shanxi 030006, China \\ ${ }^{2}$ Physics Department, Faculty of Science, Sana'a University, P.O. Box 1247, Sana"a, Yemen \\ ${ }^{3}$ Physics Department, Science \& Arts Faculty (Girls Division), Najran University, P.O. Box 1988, Najiran, Saudi Arabia \\ Correspondence should be addressed to Fu-Hu Liu; fuhuliu@163.com
}

Received 5 December 2013; Revised 16 February 2014; Accepted 24 February 2014; Published 30 March 2014

Academic Editor: Seog H. Oh

Copyright (c) 2014 Ya-Qin Gao et al. This is an open access article distributed under the Creative Commons Attribution License, which permits unrestricted use, distribution, and reproduction in any medium, provided the original work is properly cited. The publication of this article was funded by SCOAP S $^{3}$

\begin{abstract}
The double-differential $\pi^{ \pm}$production cross sections in interactions of charged pions on targets at high momentums are analyzed by using a multicomponent Erlang distribution which is obtained in the framework of a multisource thermal model. The calculated results are compared and found to be in agreement with the experimental data at the incident momentums of $3,5,8$, and $12 \mathrm{GeV} / \mathrm{c}$ measured by the HARP Collaboration. It is found that the source contributions to the mean momentum of charged particles and to the distribution width of particle momentums decrease with increase of the emission angle, and the source number and temperature do not show an obvious dependence on the emission angle of the considered particle.
\end{abstract}

\section{Introduction}

Charged particles are the main products in hadron and heavy ion induced nuclear reactions at high energies. In such collisions, the particle distributions can provide information on the properties of interacting system [1-3]. These distributions include multiplicity distribution, rapidity and pseudorapidity distributions, azimuthal and polar angular distributions, momentum and transverse momentum distributions, and double-differential cross sections.

Many models have been introduced to describe particle productions in high energy collisions. For example, the FRITIOF model [4], the VENUS model [5, 6], the RQMD model [7-9], the HIJING model [10-12], a multiphase transport model (the AMPT model) [13], the Gribov-Glauber model [14], the QGSM model [15], the color glass condensate (CGC) model [16], the perturbative QCD plus saturation plus hydrodynamics (EKRT) model [17], the ART model [18], the ZPC model $[19,20]$, the Hydrodynamics model [21, 22], the string percolation model [23], a running coupling nonlinear evolution [24], a consistent quantum mechanical multiple scattering approach (EPOS) $[25,26]$, a combination model of constituent quarks and Landau hydrodynamics [27], a twostage gluon model or a gluon dominance model [28], the KKT model [29], a multisource thermal model [30-32], and others are used in the data analyzes of high energy collisions.

As a challenging investigation, the multisource thermal model has been proposed and developed by us to describe the multiplicity distribution of charged particles [33] and isotopic production cross section of emitted fragments [34]. It is known that the multisource thermal model is very simple in describing some experimental data. Particularly, it can result in a multicomponent Erlang distribution which describes uniformly some distributions. In most cases, the experimental data have not been presented by the momentum distribution, but the transverse momentum distribution of particles. We are interested in analyzing the momentum distribution in terms of probability or double-differential 


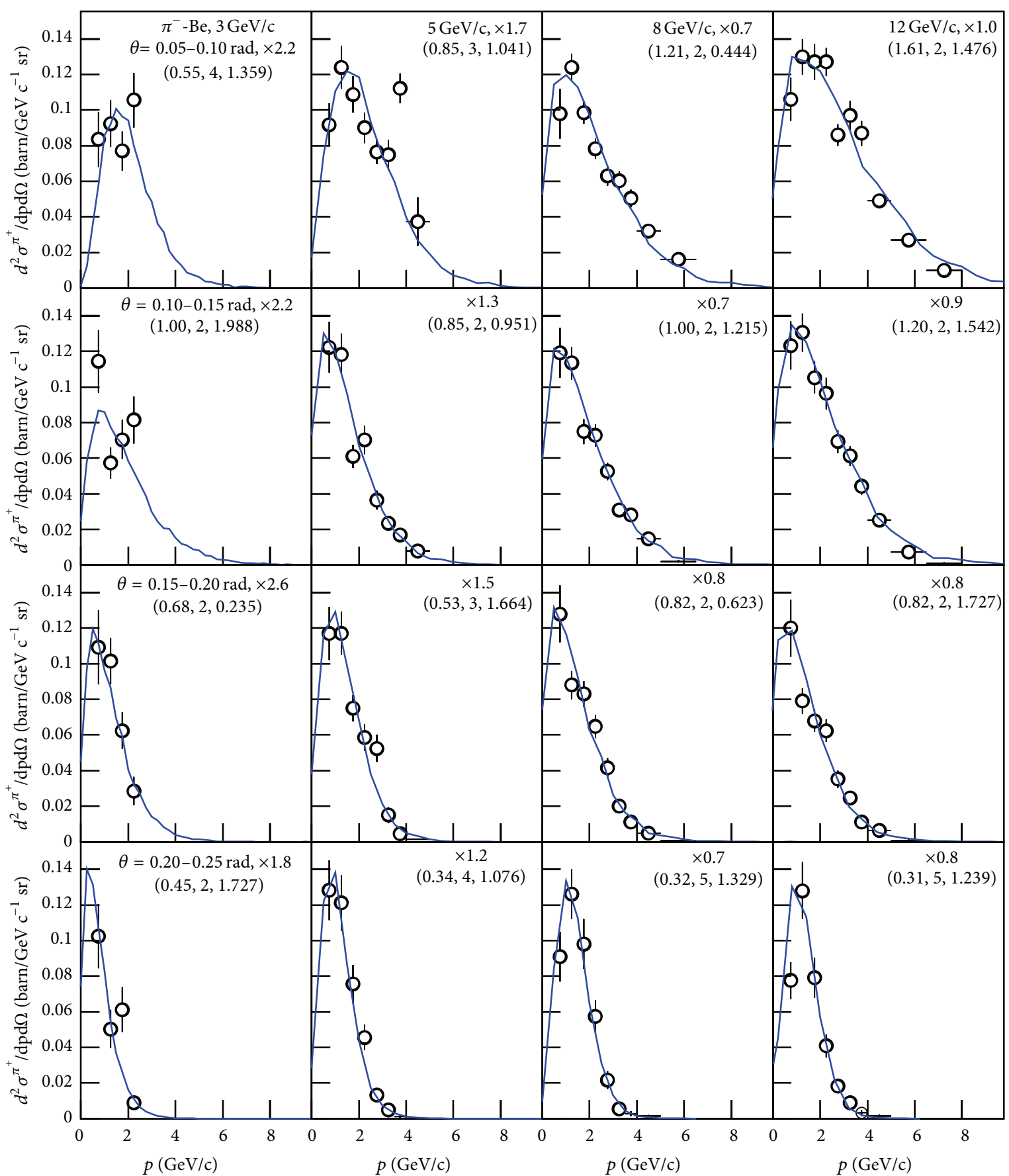

FIgURE 1: Double-differential $\pi^{+}$production cross sections in $\pi^{-}$-Be interactions at high momentums. The circles represent the experimental data of the HARP Collaboration [35] and the curves are our calculated results. Different panels are scaled by multiplying different amounts marked in the panels. The three numbers in $(\cdots)$ in each panel represent the values of $\left\langle p_{i 1}\right\rangle, m_{1}$, and $\chi^{2} /$ dof, respectively.

cross section for the further test of the multisource thermal model.

In this paper, we will use the multisource thermal model to describe the double-differential $\pi^{ \pm}$production cross sections in charged pion induced nuclear reactions at high momentums. The model is described in Section 2. The comparisons with the experimental data at the incident momentums of $3,5,8$, and $12 \mathrm{GeV} / \mathrm{c}$ measured by the HARP
Collaboration [35] are shown in Section 3. Finally, we give our conclusions in Section 4.

\section{The Model and Method}

The model used in the present work can be found in our previous work $[33,34]$ which present related formulations 


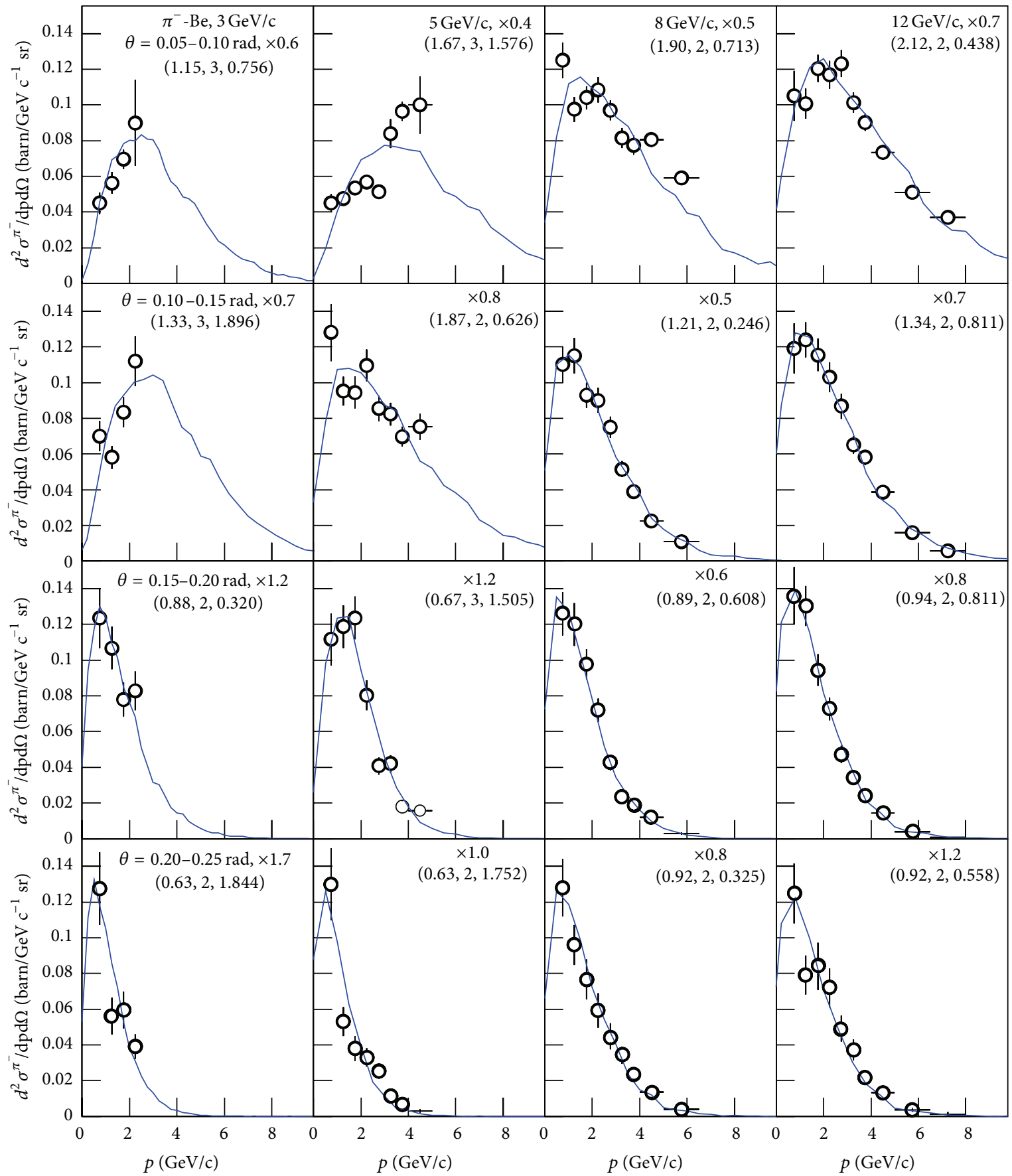

FIgURE 2: The same as Figure 1, but showing the results for $\pi^{-}$production cross sections.

in terms of multiplicity distribution of final-state products and neutron number distribution in isotopes. To give a whole presentation of the present work, we introduce briefly the model $[33,34]$ on the multicomponent Erlang distribution in terms of charged particle momentum $p$ and its distribution in the following. In the model, many emission sources of particles and fragments are assumed to form in collisions. According to different interaction mechanisms or event samples, the sources are divided into $l$ groups (subsamples). The source number in the $j$ th group is assumed to be $m_{j}$.
It is assumed that each source contributes to momentum distribution to be an exponential function, that is, the momentum $\left(p_{i j}\right)$ distribution contributed by the $i$ th source in the $j$ th group is given by

$$
f_{i j}\left(p_{i j}\right)=\frac{1}{\left\langle p_{i j}\right\rangle} \exp \left(-\frac{p_{i j}}{\left\langle p_{i j}\right\rangle}\right),
$$

where $\left\langle p_{i j}\right\rangle$ denotes the mean momentum contributed by the $i$ th source in the $j$ th group $[33,34]$. Generally, the mean 


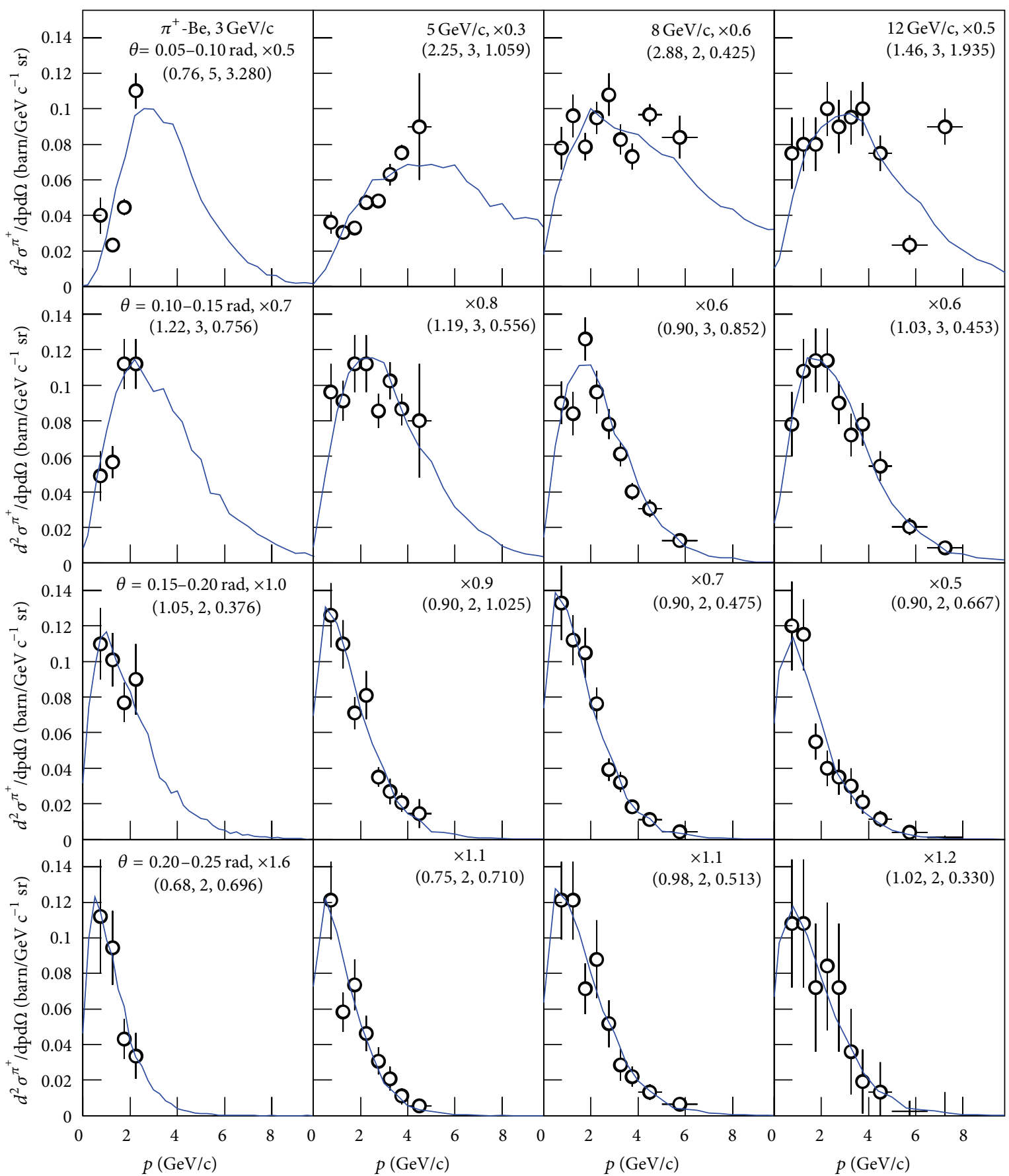

Figure 3: The same as Figure 1, but showing the results in $\pi^{+}$-Be interactions.

momentum contributed by different sources in the same group is assumed to be the same. The particle momentum distribution contributed by the $j$ th group is then given by the folding of $m_{j}$ exponential functions [33, 34]:

$$
f_{j}(p)=\frac{p^{m_{j}-1}}{\left(m_{j}-1\right) !\left\langle p_{i j}\right\rangle^{m_{j}}} \exp \left(-\frac{p}{\left\langle p_{i j}\right\rangle}\right) .
$$

One can see that an Erlang distribution is obtained. The momentum distribution contributed by the $l$ groups is given by a weighted sum of $l$ Erlang distributions:

$$
f(p)=\sum_{j=1}^{l} k_{j} f_{j}(p),
$$

where $k_{j}$ denotes the weight factor and obeys the normalization $\sum_{j=1}^{l} k_{j}=1$. To avoid the factorial calculation in (2) in 


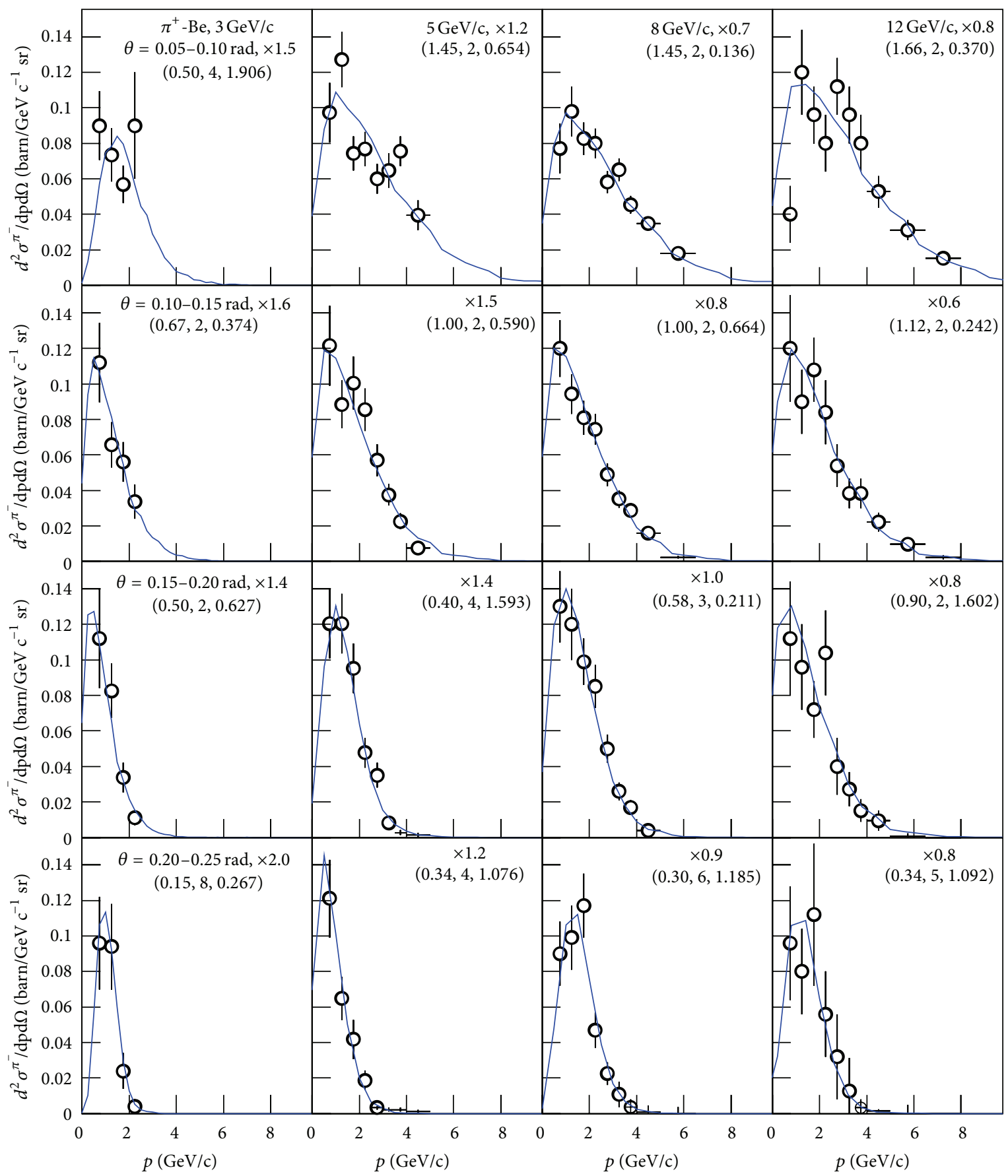

FIGURE 4: The same as Figure 1, but showing the results for $\pi^{-}$production cross sections in $\pi^{+}$- Be interactions.

the case of $m_{j}$ being a large value, the Monte Carlo method is used to calculate the momentum distribution $[33,34]$.

In the Monte Carlo calculation, let $R_{i j}$ denote random variable in $[0,1]$. We have

$$
p_{i j}=-\left\langle p_{i j}\right\rangle \ln R_{i j}
$$

for the $i$ th source in the $j$ th group due to (1) which obeys the exponential function. The momentum contributed by the $j$ th group is

$$
p=-\sum_{i=1}^{m_{j}}\left\langle p_{i j}\right\rangle \ln R_{i j}
$$




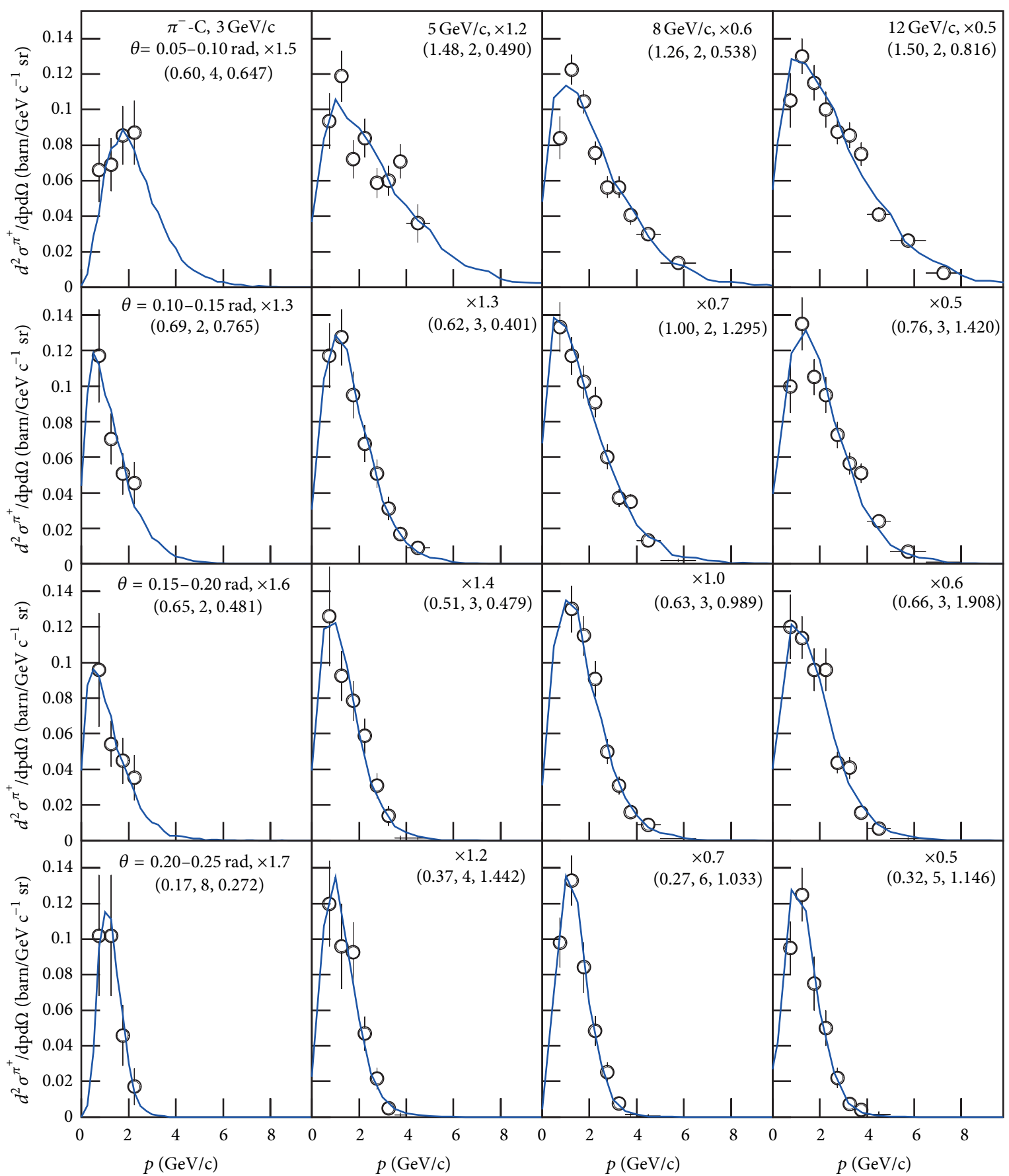

Figure 5: The same as Figure 1, but showing the results for $\pi^{+}$production cross sections in $\pi^{-}$-C interactions.

due to the fact that (2) is the folding of $m_{j}$ exponential functions. The mean momentum contributed by the $l$ groups is

$$
\langle p\rangle=\sum_{j=1}^{l} k_{j}\left\langle p_{i j}\right\rangle m_{j}
$$

due to the fact that (3) is a weighted sum.
To obtain the parameter values, for the purpose of convenience, we can use the idea of the least-square method. The values of $\left\langle p_{i j}\right\rangle$ and $m_{j}$ are changed from low to high step by step and the values of $\chi^{2}$ can be obtained. The former parameter can change continuously and the latter one is a series of integers. The best parameter values correspond to the minimum $\chi^{2}$ value, and the acceptable $\chi^{2}$ values determinate the parameter errors. 


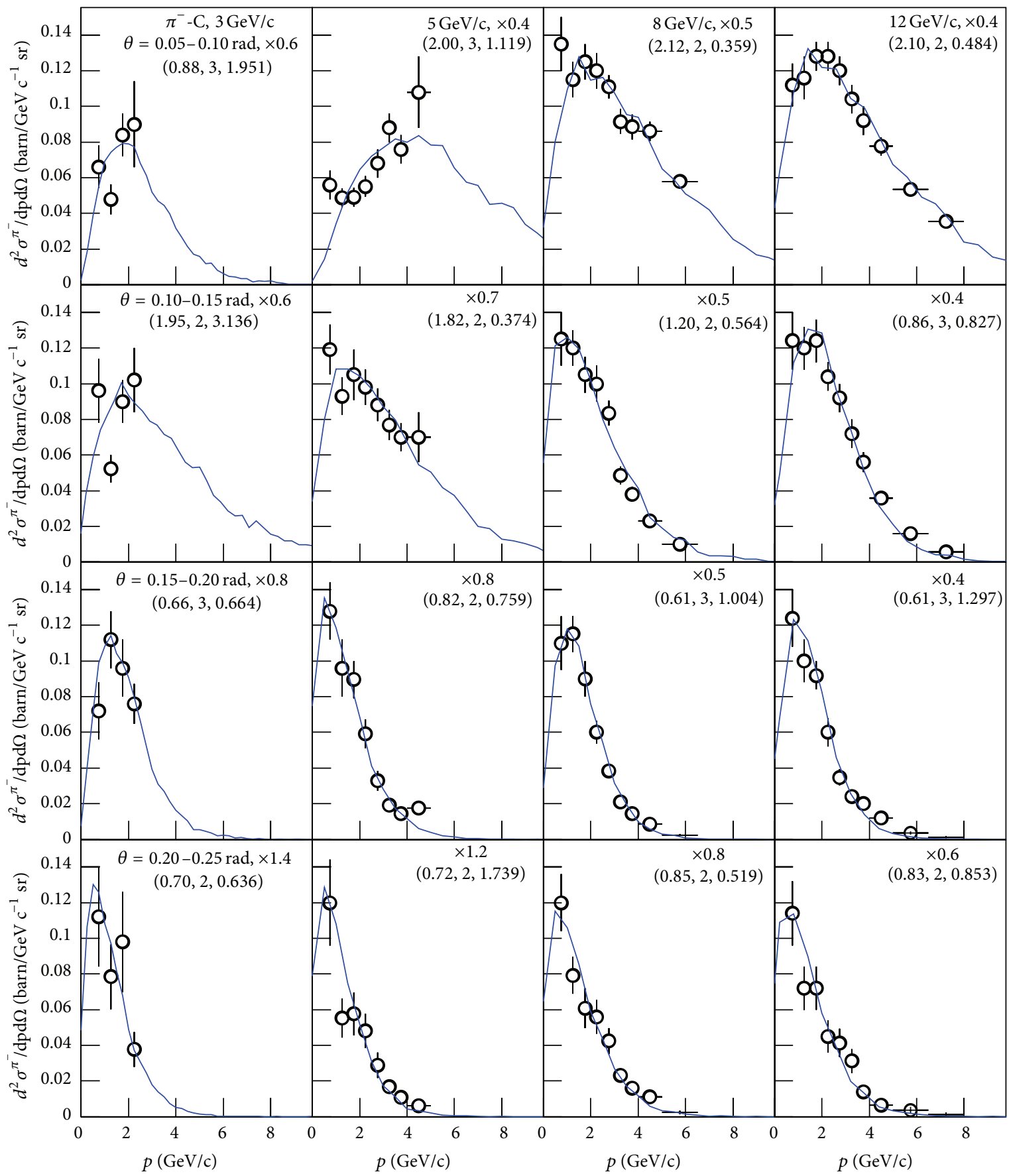

Figure 6: The same as Figure 1, but showing the results for $\pi^{-}$production cross sections in $\pi^{-}$-C interactions.

In the model, the value of $l$ denotes the number of types of emission sources. Generally, $l=1-3$, which renders a small number of types of emission sources. The total number of emission sources can be small or large. A small number of emission sources means the hadronic sources, and a large number of emission sources means the parton degree of freedom. In the charged pion induced nuclear reactions at 3$12 \mathrm{GeV} / \mathrm{c}$ which are considered in the present work, we expect a small number of emission sources due to the saturation effect of target nuclei and not too high incident momentums.

The imbalances in mechanics and geometry render the interacting system to have kinetic, hydrodynamical, and thermaldynamical evolutions. The system or subsystems are assumed to stay in an equilibrium state or in local equilibrium states at the stage of chemical freeze-out. In the model, the inverse slope (mean transverse momentum) 


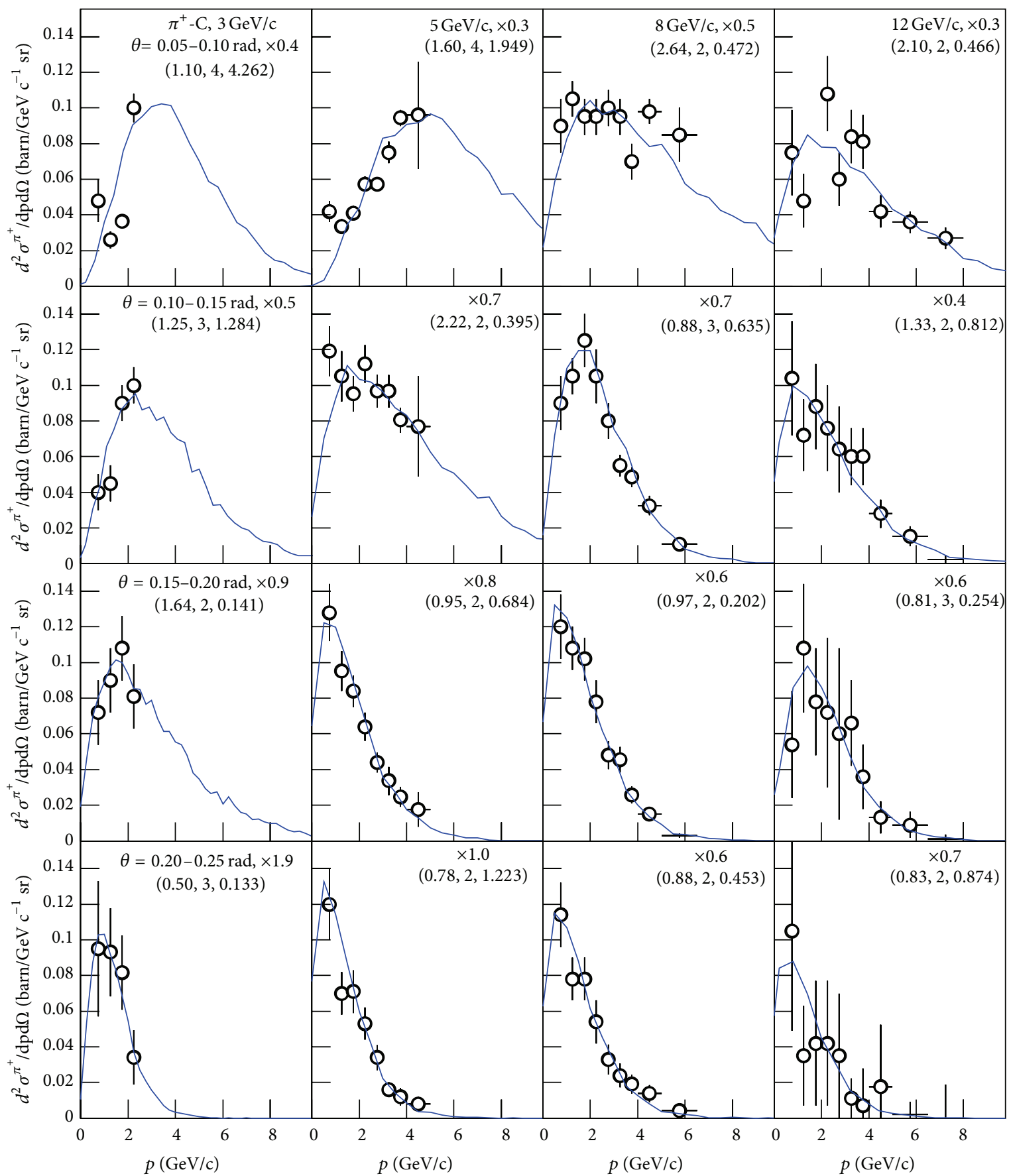

FIgURE 7: The same as Figure 1, but showing the results for $\pi^{+}$production cross sections in $\pi^{+}$-C interactions.

in the exponential distribution of transverse momentums is approximately regarded as the temperature parameter. According to the relation between the transverse momentum $p_{T}$ and the momentum $p$, we have the temperature $T \approx$ $\sum_{j=1}^{l} k_{j}\left\langle p_{i j}\right\rangle \sin \theta$ for a given polar angle $\theta$. Although there is a $\theta$ in the presentation of $T$, we do not expect that there is a dependence of $T$ on $\theta$.

\section{Comparisons with Experimental Data}

The double-differential $\pi^{+}$and $\pi^{-}$production cross sections measured in different emission angle $(\theta)$ ranges in $\pi^{-}$-Be interactions at $3,5,8$, and $12 \mathrm{GeV} / \mathrm{c}$ are shown in Figures 1 and 2, respectively. Different panels are scaled by multiplying different amounts to give all of them in a whole figure. The circles represent the experimental data of the HARP 


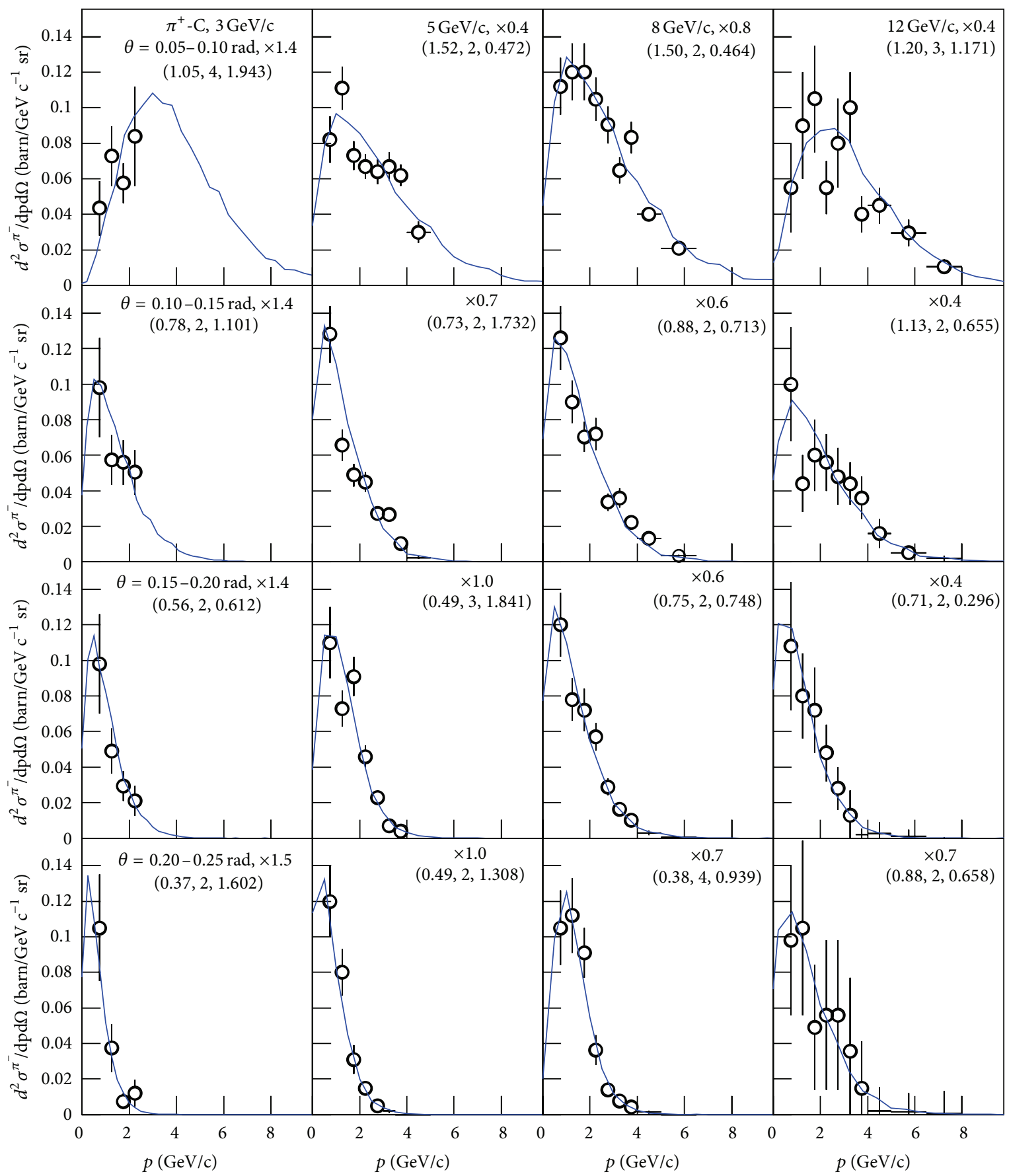

FIgURE 8: The same as Figure 1, but showing the results for $\pi^{-}$production cross sections in $\pi^{+}$-C interactions.

Collaboration [35] and the curves are our calculated results. In the calculation, we have used $l=1$. The best values of $\left\langle p_{i 1}\right\rangle$ and $m_{1}$, as well as the value of $\chi^{2}$ per degree of freedom $\left(\chi^{2} / \mathrm{dof}\right)$, are given in the figure in terms of $\left(\left\langle p_{i 1}\right\rangle\right.$, $m_{1}, \chi^{2} /$ dof $)$, where $\left\langle p_{i 1}\right\rangle$ is in the units of $\mathrm{GeV} / \mathrm{c}$. The relative errors for $\left\langle p_{i 1}\right\rangle$ are about $6 \%$, which are only statistical errors, and the systematic errors are eliminated. The error for $m_{1}$ can be neglected due to that the smallest alteration $m_{1} \pm 1$ results in different distribution shape with an unexpected large $\chi^{2}$.
Similarly, the double-differential $\pi^{+}$and $\pi^{-}$production cross sections measured in different $\theta$ ranges in $\pi^{+}$-Be interactions at 3, 5, 8, and $12 \mathrm{GeV} / \mathrm{c}$ are shown in Figures 3 and 4, respectively. From Figures 1-4 one can see that the model with $l=1$ describes uniformly the experimental data in most cases.

Figures 5-8 are the same as Figures 1-4, respectively, but showing the results for $\pi^{\mp}-\mathrm{C}$ interactions. Figures 9-12 are the same as Figures 1-4, respectively, too, but showing 


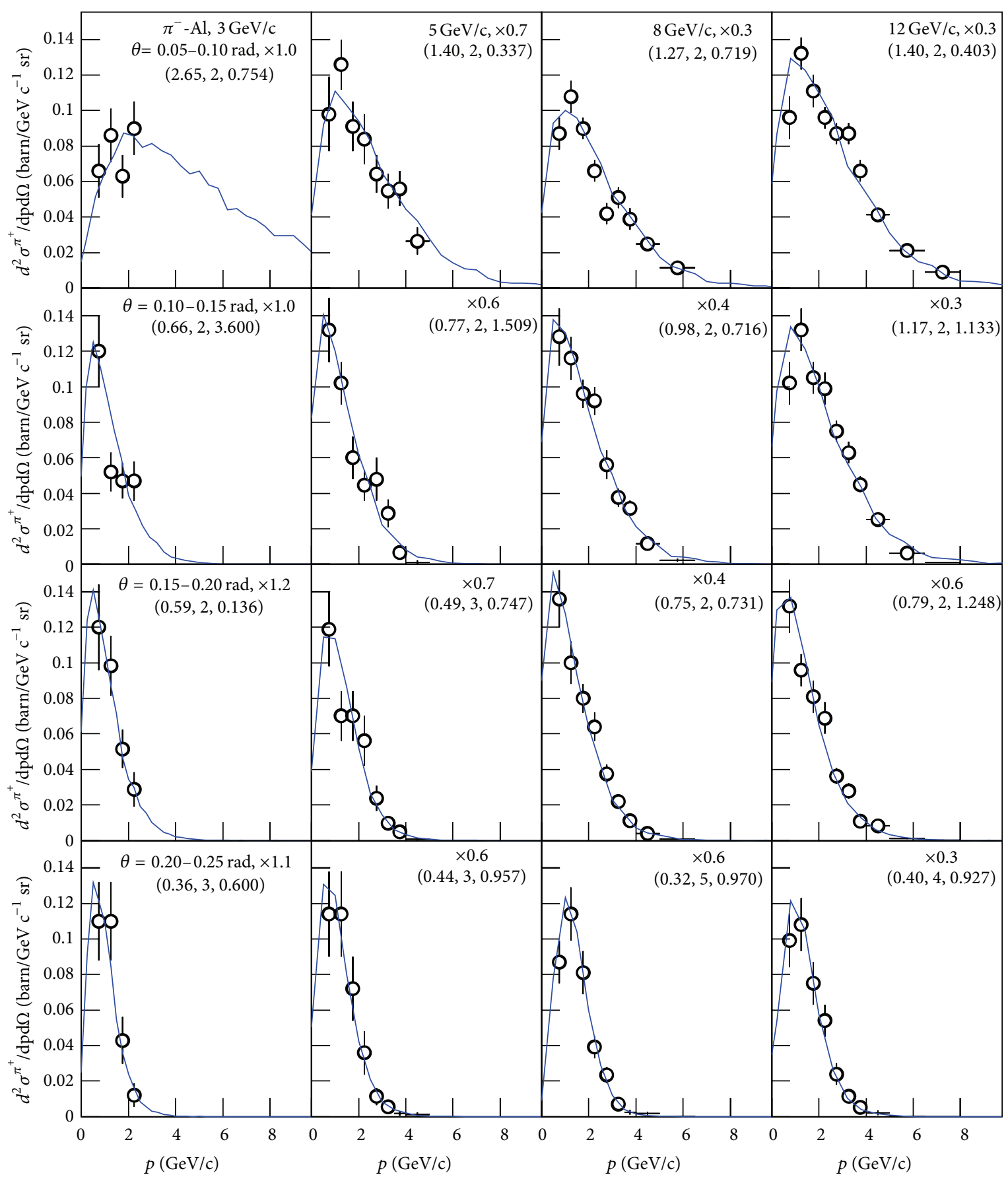

FIGURE 9: The same as Figure 1, but showing the results for $\pi^{+}$production cross sections in $\pi^{-}$-Al interactions.

the results for $\pi^{\mp}-\mathrm{Al}$ interactions. We see that the model describes uniformly the experimental data in most cases. In [35], the experimental data on $\pi^{\mp}-\mathrm{Cu}, \mathrm{Sn}, \mathrm{Ta}$, and $\mathrm{Pb}$ interactions at high momentums have been given, too. A similar conclusion can be obtained in the case of fitting the experimental data by using the model.

From Figures 1-12 we see that the distribution width which is represented by $\left\langle p_{i 1}\right\rangle m_{1}$ shows a decrease with increases of $\theta$. This phenomenon renders that the emission source has a forward movement along the beam direction.
As a result, comparing with the situation in the source rest frame, the pion with small $\theta$ has a large momentum and that with large $\theta$ has a small momentum due to the effect of source momentum. We see also from Figures 1-12 that $m_{1}$ is only in the range from 2 to 12 and the most probability is 2 . The sources are obviously incident pion and target nucleons. The number of participant target nucleons is in the range from 1 to 11 and the most probability is 1 . Except the participant nucleons, other nucleons in target nucleus are the spectator nucleons. 


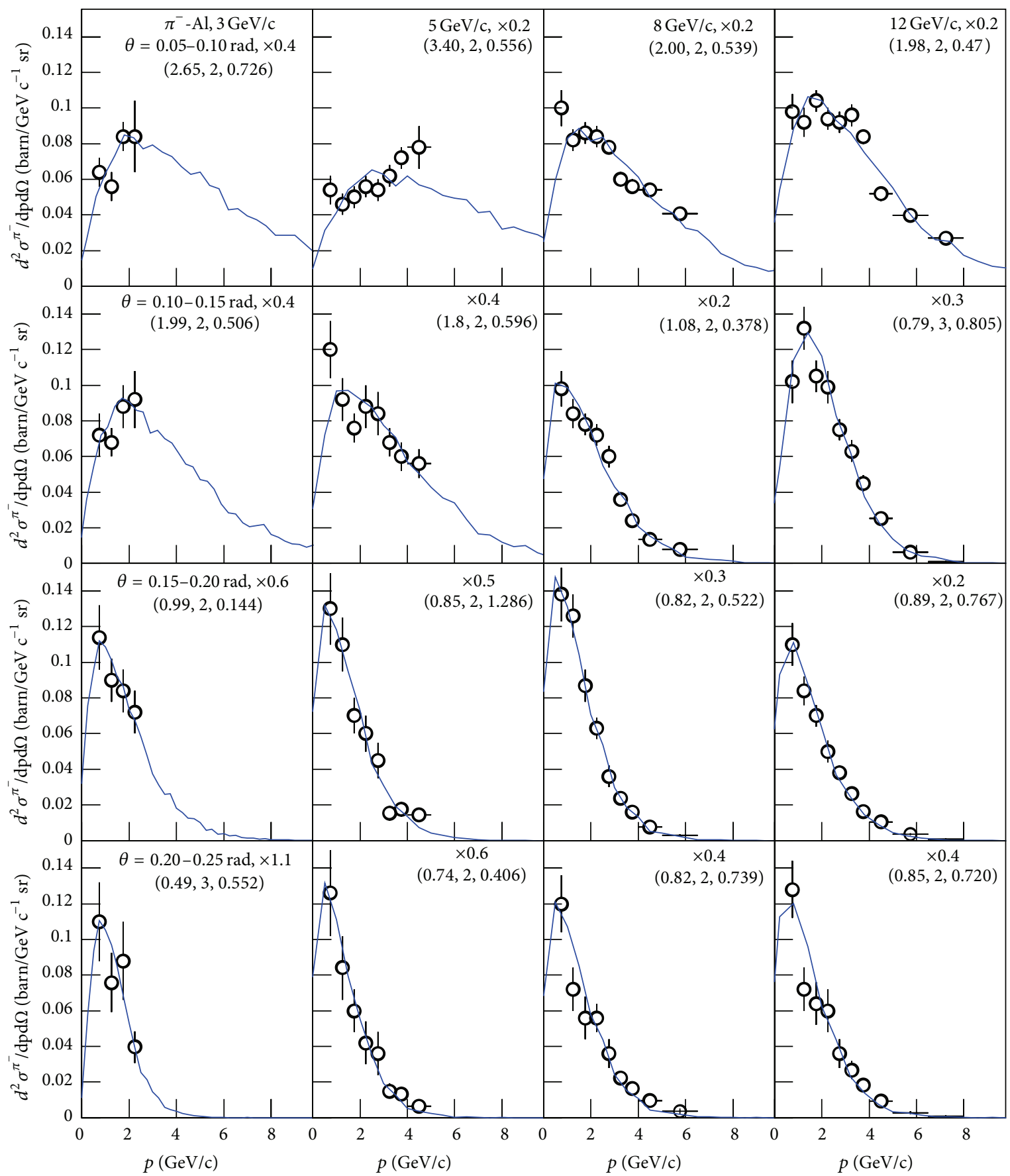

FIgURE 10: The same as Figure 1, but showing the results for $\pi^{-}$production cross sections in $\pi^{-}$-Al interactions.

To see clearly the changing trends of the parameters, the dependences of $\left\langle p_{i 1}\right\rangle, m_{1},\left\langle p_{i 1}\right\rangle m_{1}$, and $T$ on $\theta$ for $\pi^{+}$produced in $\pi^{-}-\mathrm{Be}, \mathrm{C}$, and $\mathrm{Al}$ interactions are shown in Figure 13. The different symbols represent the parameter values obtained at different momentums, and for targets Be, $\mathrm{C}$, and $\mathrm{Al}$, the results are taken from Figures 1, 5, and 9, respectively. Similarly, the corresponding dependences for $\pi^{-}$produced in the same interactions are given in Figure 14 which holds the parameter values taking from Figures 2, 6, and 10. The results for $\pi^{+}$and $\pi^{-}$produced in $\pi^{+}$induced interactions are presented in Figures 15 and 16 which hold the parameter values taking from Figures 3, 7, and 11, as well as Figures 4,8 , and 12, respectively. We see that $\left\langle p_{i 1}\right\rangle$ has a decreasing trend, $m_{1}$ and $T$ do not show an obvious change, and $\left\langle p_{i 1}\right\rangle m_{1}$ has a decreasing trend with increasing $\theta$. This renders again that the emission source has a forward movement along the beam direction, which leads the momentum distribution width of produced pions to be small at large $\theta$. The target nucleus presents a saturation effect on the source number and temperature. 


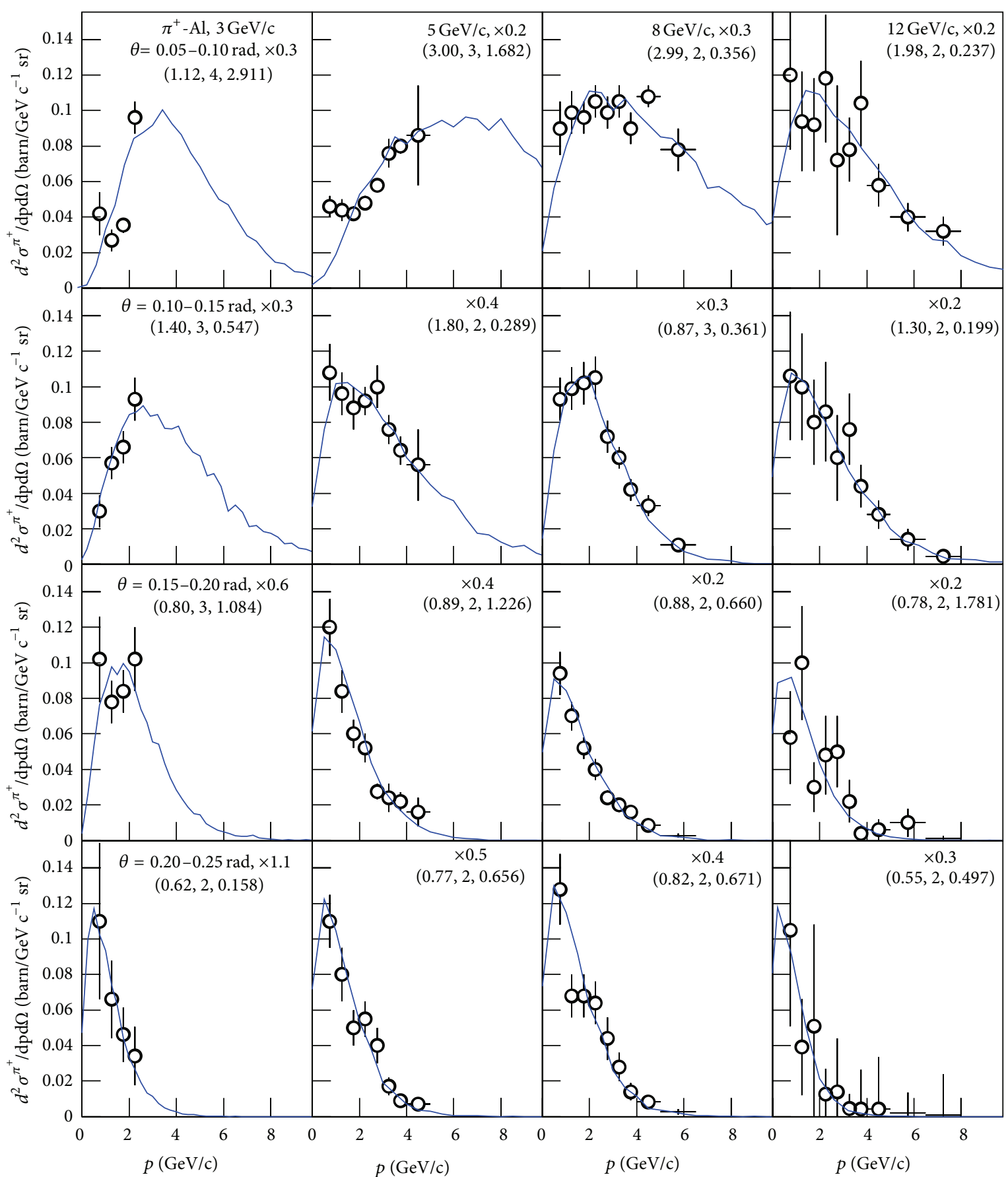

FIGURE 11: The same as Figure 1, but showing the results for $\pi^{+}$production cross sections in $\pi^{+}-\mathrm{Al}$ interactions.

From Figures 1-16, we see that the changing trends of the parameters on incident momentum and target size are not obvious. We have not seen an obvious difference of parameters for $\pi^{+}$and $\pi^{-}$produced in reactions induced by incident $\pi^{+}$and $\pi^{-}$. To see clearly the dependences of parameters on incident momentum $(P)$ and target size, we combine the results of $\pi^{ \pm}$produced in the four $\theta$ ranges in reactions induced by $\pi^{ \pm}$and give the mean values of $\left\langle p_{i 1}\right\rangle, m_{1}$, $\left\langle p_{i 1}\right\rangle m_{1}$, and $T$ (i.e., $\overline{\left\langle p_{i 1}\right\rangle}, \overline{m_{1}}, \overline{\left\langle p_{i 1}\right\rangle m_{1}}$, and $\bar{T}$ ) for different incident momentums and target nuclei in Figures 17(a)$17(\mathrm{~d})$, respectively, where the error bars for $\overline{\left\langle p_{i 1}\right\rangle}, \overline{\left\langle p_{i 1}\right\rangle m_{1}}$, and $\bar{T}$ are obtained according to the error transformation formula, and those for $\overline{m_{1}}$ are standard deviations. One can see that the concerned mean values have no obvious dependence on incident momentum and target size. We would like to point out that the nondependence on incident 


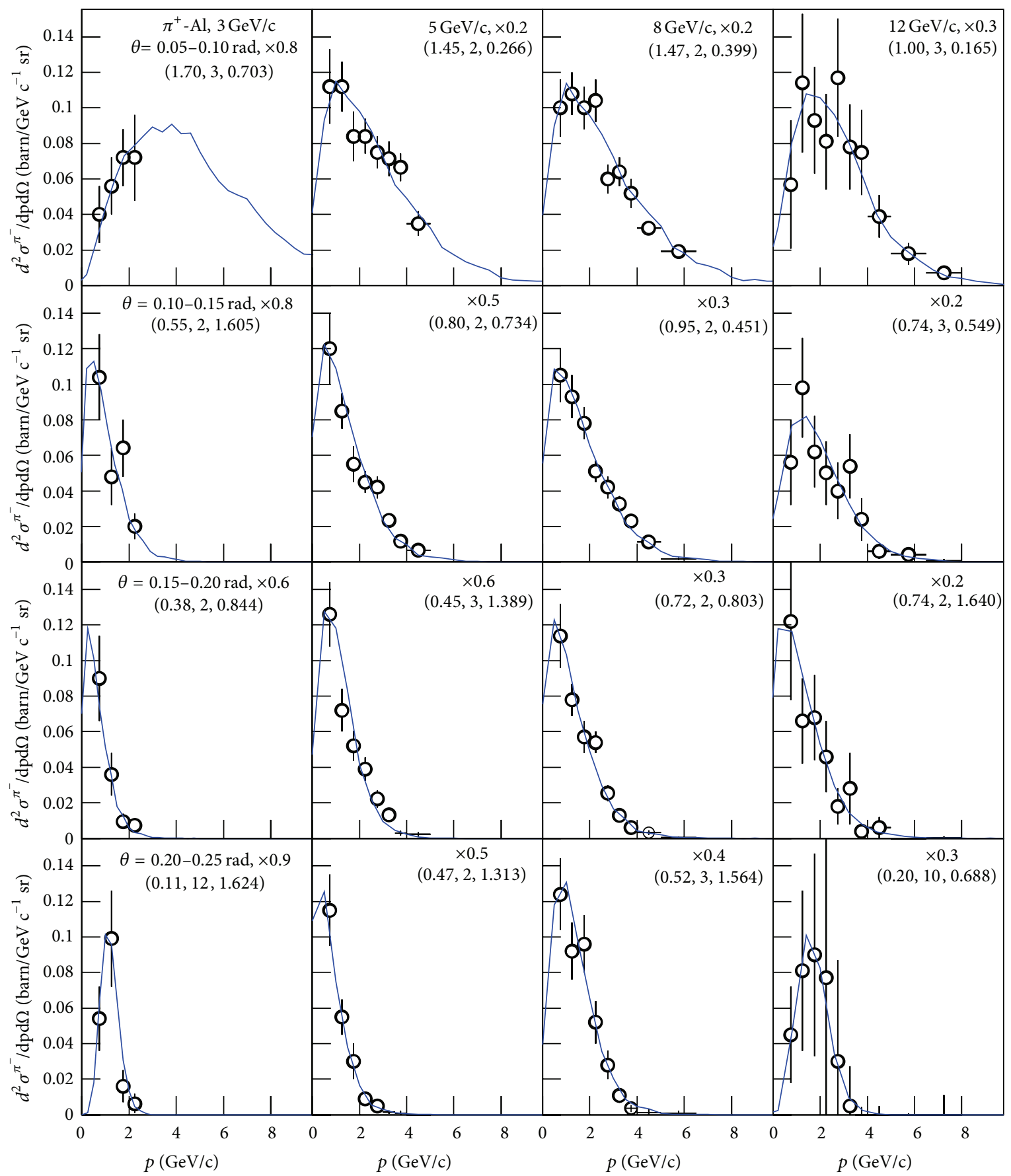

FIGURE 12: The same as Figure 1, but showing the results for $\pi^{-}$production cross sections in $\pi^{+}-\mathrm{Al}$ interactions.

momentum may be a result of the too narrow range of incident momentums, and the nondependence on target size renders the saturation effect of target nuclei in charged pion induced reactions at the considered incident momentums. Particularly, the extracted mean temperatures are in the range from $(107 \pm 7) \mathrm{MeV}$ to $(147 \pm 9) \mathrm{MeV}$ which are lower than the critical temperature $(156 \mathrm{MeV})$ for phase transition from hadron matter to quark matter obtained by the thermaldynamic model [36].

\section{Conclusions and Discussions}

To conclude, the multisource thermal model is used to give a new and simple description of the double-differential $\pi^{ \pm}$ production cross sections measured in different emission angle ranges in interactions of $\pi^{\mp}$ on different targets at the considered incident momentums. In most cases, the model describes well the experimental data of the HARP Collaboration. In the calculation we have used $l=1$. This 


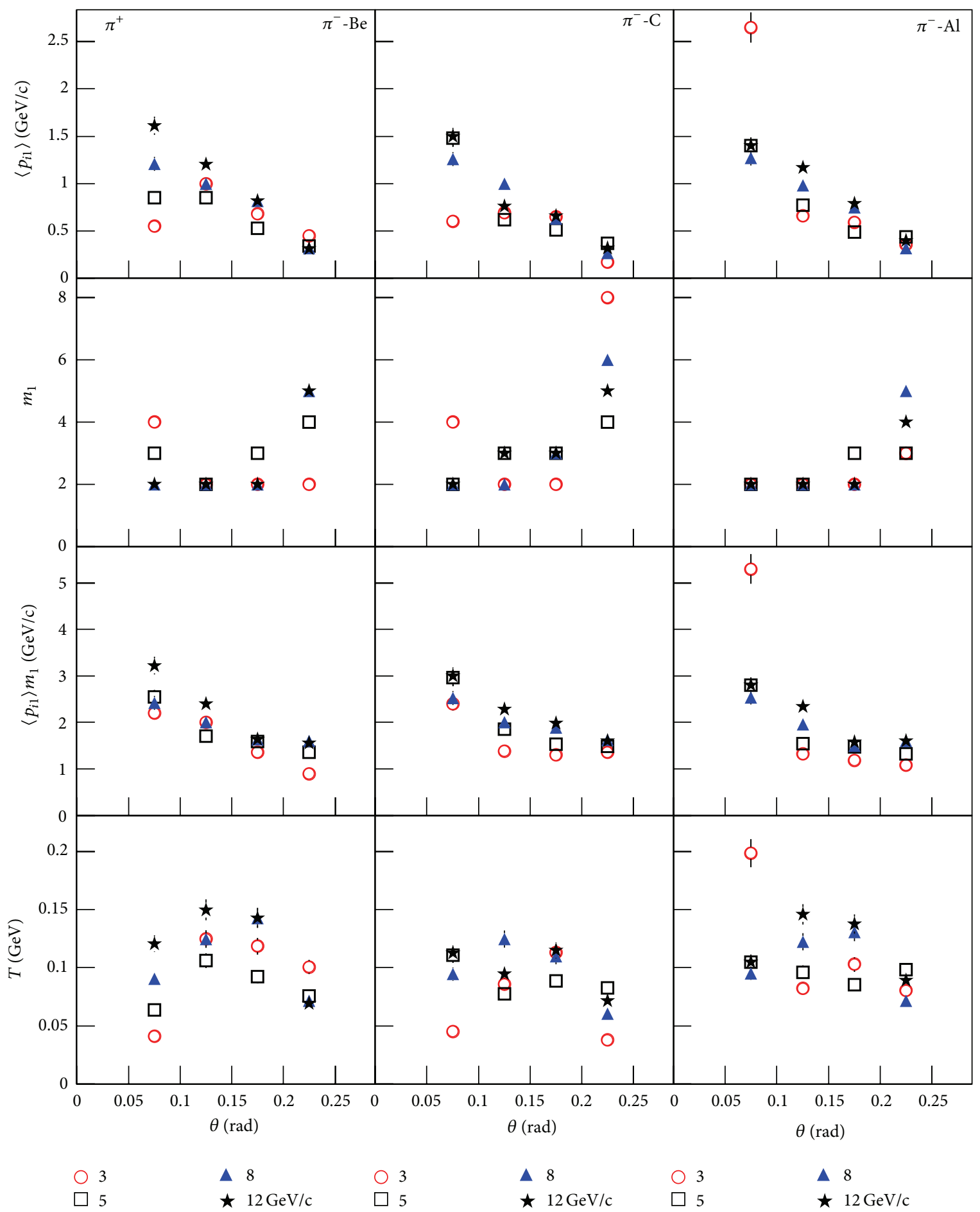

FIGURE 13: Dependences of $\left\langle p_{i 1}\right\rangle, m_{1},\left\langle p_{i 1}\right\rangle m_{1}$, and $T$ on $\theta$ for $\pi^{+}$produced in $\pi^{-}$-Be, $\mathrm{C}$, and $\mathrm{Al}$ interactions. The different symbols represent the parameter values obtained at different momentums. The parameter values for targets $\mathrm{Be}, \mathrm{C}$, and $\mathrm{Al}$ are taken from Figures 1,5 , and 9 , respectively.

means that there is only one type of emission sources in the considered emission angle ranges. The parameter $m_{1}$ is small, which renders that the emission sources are incident pion and target nucleons. The structures of pion and nucleon do not play an important role at the considered incident momentums.

The parameter $m_{1}$ does not show an obvious change with increases of $\theta$. For a given interaction, it is a natural result that 


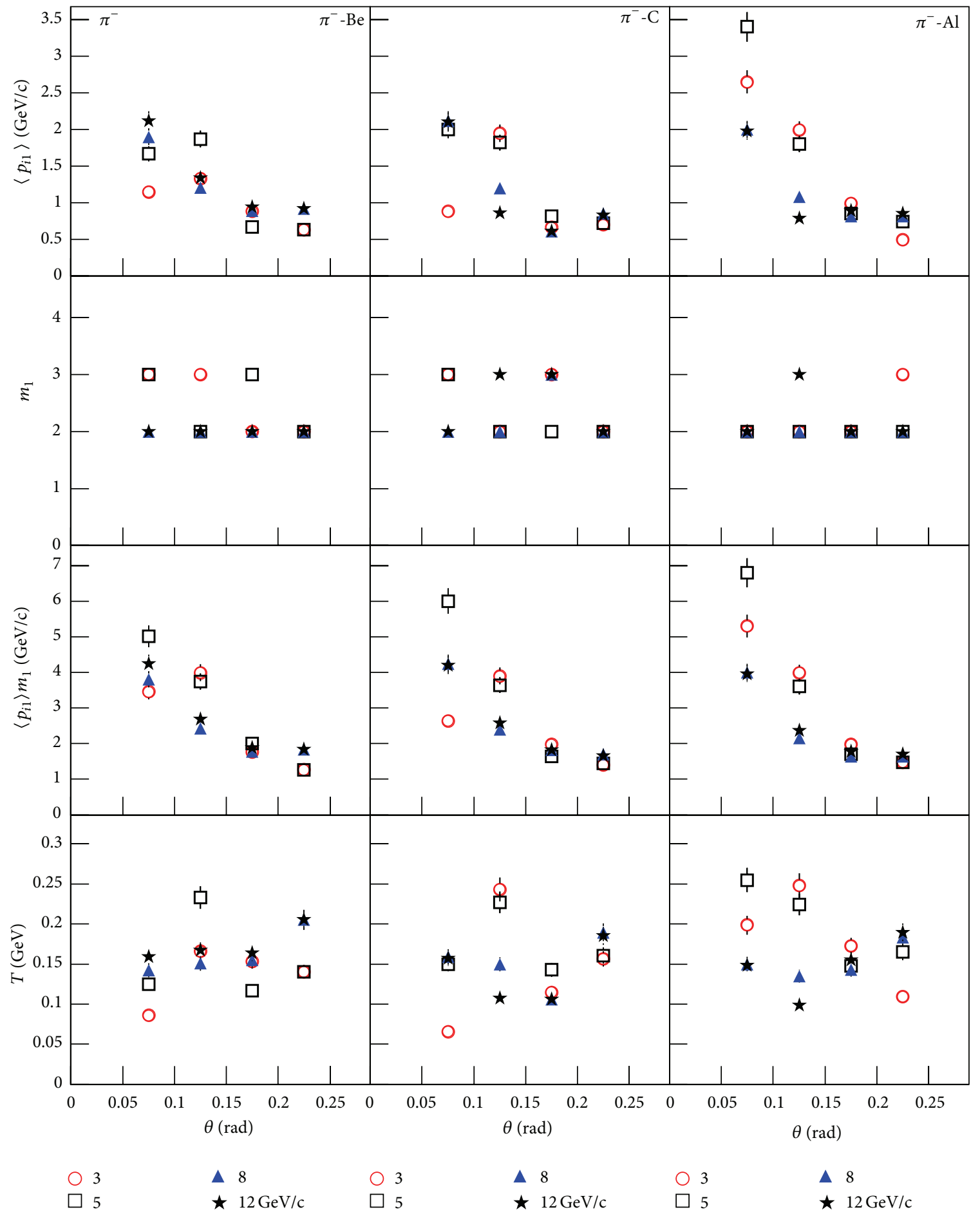

FIGURE 14: The same as Figure 13, but showing the results for $\pi^{-}$. The parameter values for targets Be, C, and $\mathrm{Al}$ are taken from Figures 2,6 , and 10 , respectively.

the source number has no relation with the emission angle of produced particle. The parameter $\left\langle p_{i 1}\right\rangle$ and the product $\left\langle p_{i 1}\right\rangle m_{1}$ decrease obviously with increases of $\theta$. Considering the noneffect of $m_{1},\left\langle p_{i 1}\right\rangle$ has the same behavior as $\left\langle p_{i 1}\right\rangle m_{1}$. As the distribution width of particle momentum, $\left\langle p_{i 1}\right\rangle m_{1}$ has a small value at large $\theta$ because the emission source has a forward movement along the beam direction and the particle has a momentum transformation from the source rest frame to the laboratory reference frame.

As the source number, $m_{1}$ is not related to the concerned incident momentum and target size. The source contribution $\left(\left\langle p_{i 1}\right\rangle\right)$ to the mean momentum of charged particles and 


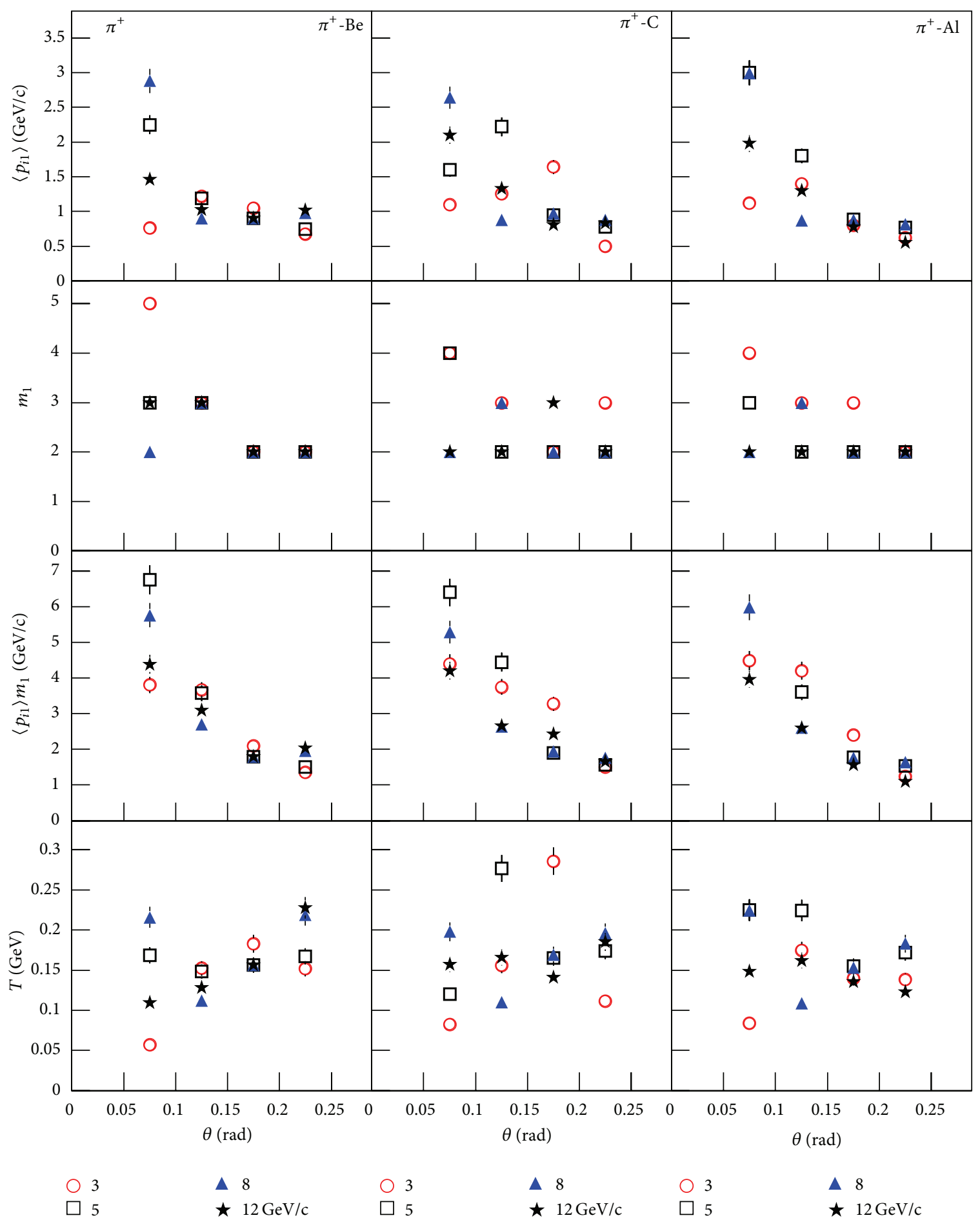

FIGURE 15: The same as Figure 13, but showing the results for $\pi^{+}$produced in $\pi^{+}-\mathrm{Be}, \mathrm{C}$, and $\mathrm{Al}$ interactions. The parameter values for targets $\mathrm{Be}, \mathrm{C}$, and $\mathrm{Al}$ are taken from Figures 3, 7, and 11, respectively.

the distribution width $\left(\left\langle p_{i 1}\right\rangle m_{1}\right)$ of the particle momentums are not related to the concerned incident momentum and target size, too. The nonrelation to the concerned incident momentum is caused by the narrow momentum range, and the nonrelation to the target size is the result of saturation effect of target nuclei in the concerned reactions. It is expected that the concerned parameters will increase at a high enough incident momentum.

The extracted temperatures do not show an obvious dependence on the incident momentum, target size, and 


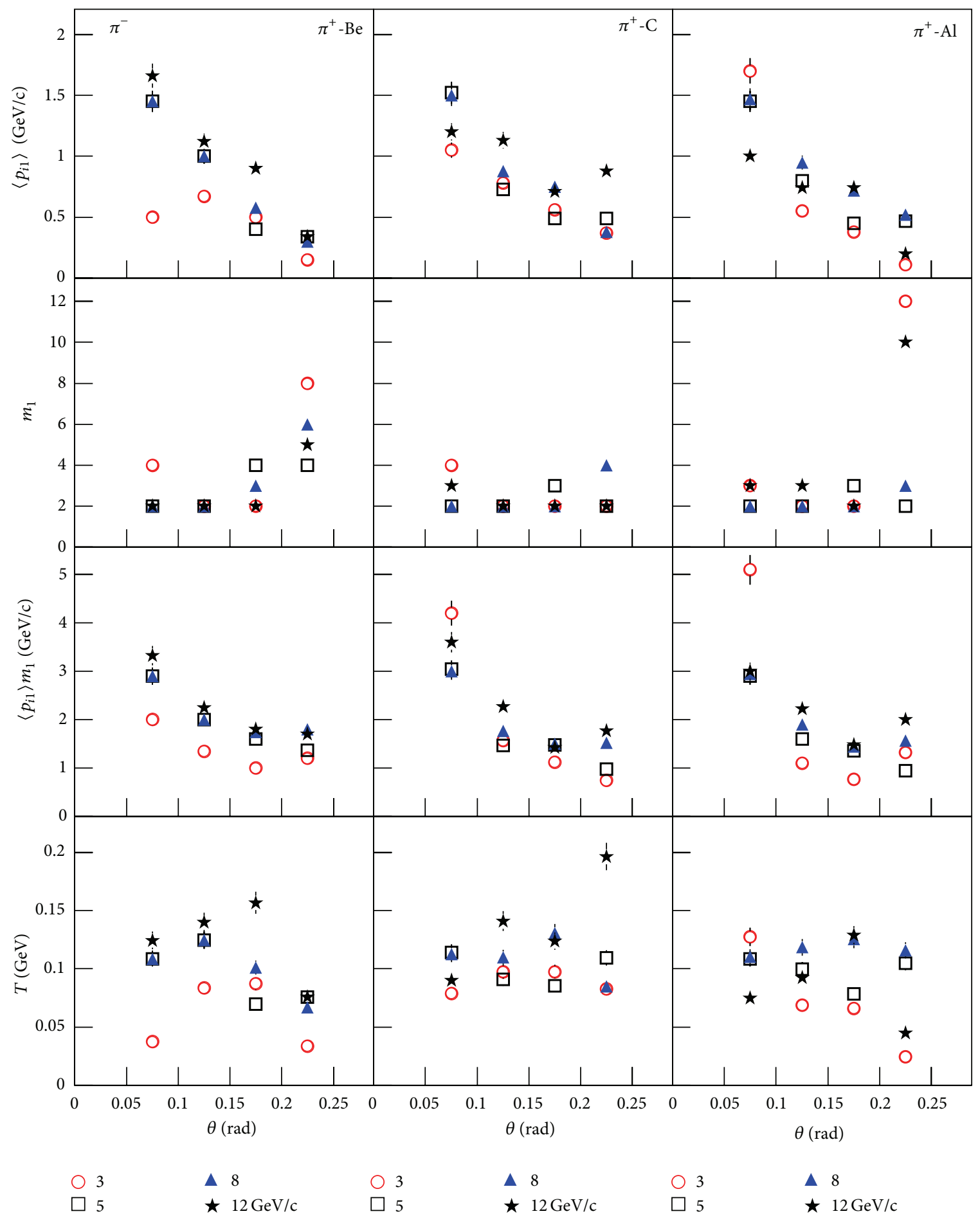

FIGURE 16: The same as Figure 13, but showing the results for $\pi^{-}$produced in $\pi^{+}-\mathrm{Be}, \mathrm{C}$, and $\mathrm{Al}$ interactions. The parameter values for targets $\mathrm{Be}, \mathrm{C}$, and $\mathrm{Al}$ are taken from Figures 4,8 , and 12 , respectively.

polar angle in the considered collisions. The target nucleus presents a saturation effect on the source temperature. The extracted mean temperatures over polar angles are in the range from $(107 \pm 7) \mathrm{MeV}$ to $(147 \pm 9) \mathrm{MeV}$ which are lower than the critical temperature $(156 \mathrm{MeV})$ for phase transition from hadron matter to quark matter obtained by the thermaldynamic model [36]. It is expected that higher temperatures will be obtained at higher incident momentums.

Because the transverse momentum $p_{T}=p \sin \theta$, the distribution of $p_{T}$ is similar to that of $p$ at a given $\theta$. 


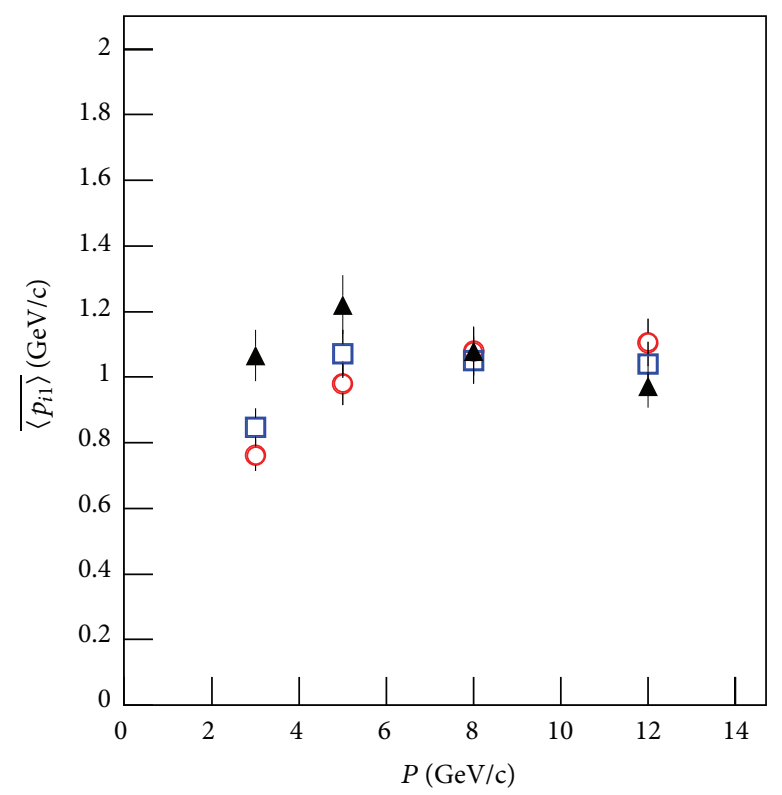

(a)

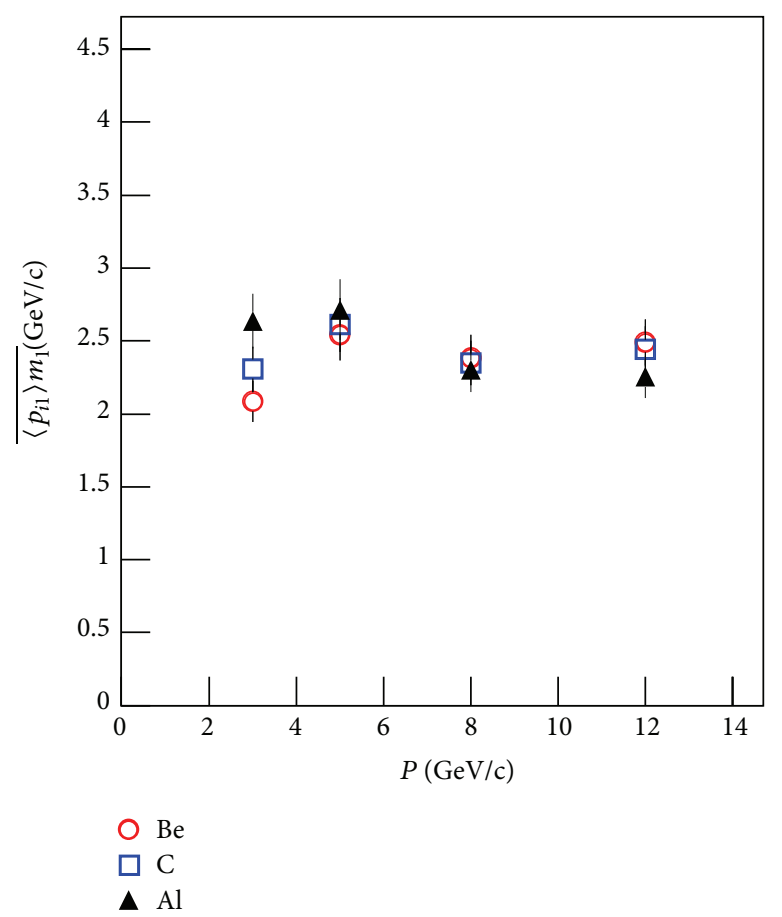

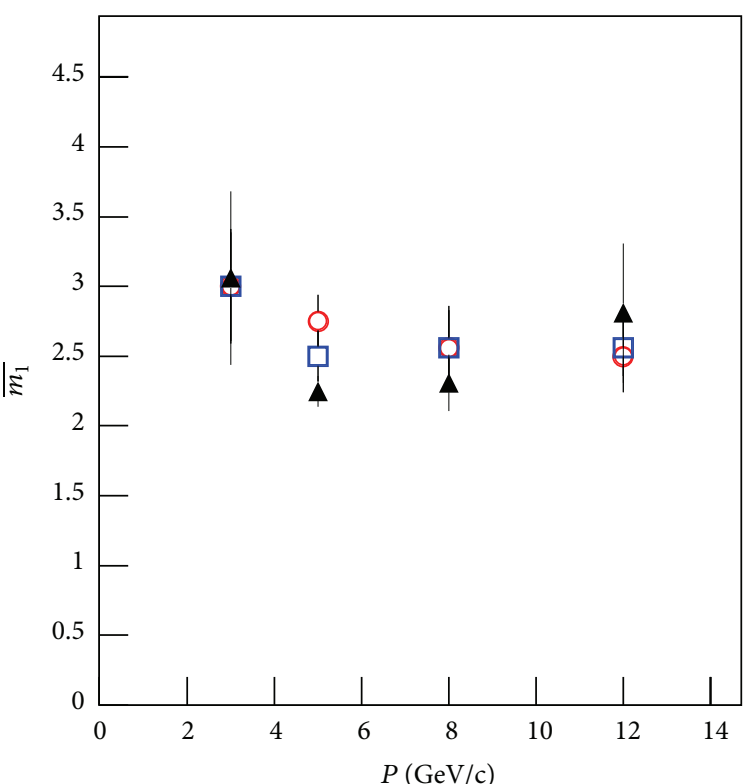

(b)

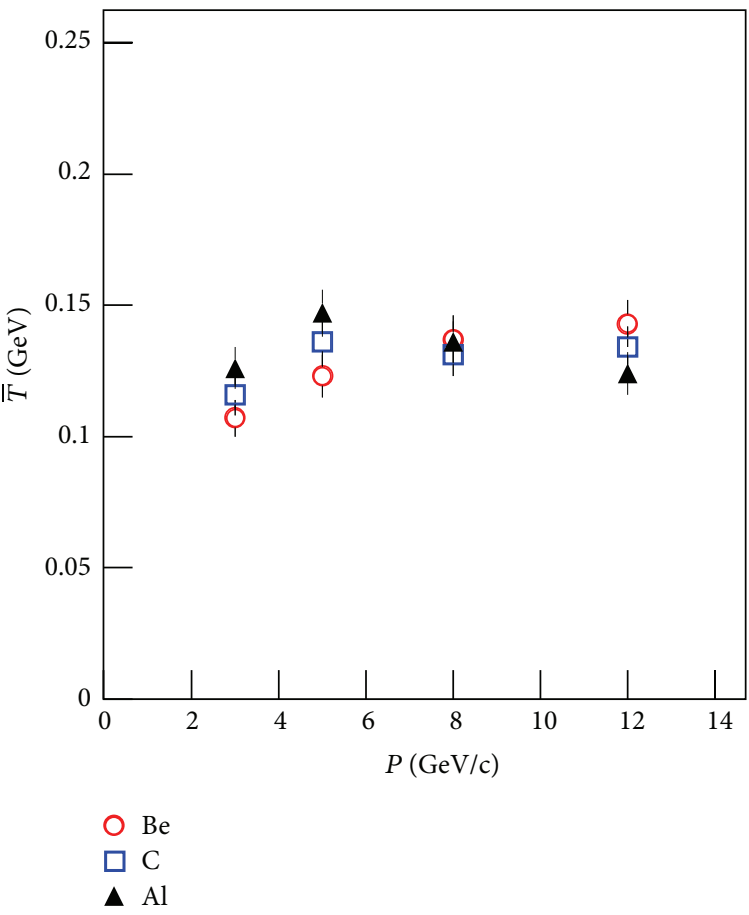

(d)

FIGURE 17: Dependences of (a) $\overline{\left\langle p_{i 1}\right\rangle}$, (b) $\overline{m_{1}}$, (c) $\overline{\left\langle p_{i 1}\right\rangle m_{1}}$, and (d) $\bar{T}$ on incident momentum $P$ for $\pi^{ \pm}$produced in $\pi^{ \pm}$induced reactions. The different symbols represent the corresponding values obtained for different targets. The original values of the concerned parameters are taken from Figures 1-12.

The present modelling description can be used for the transverse momentum distribution if we use $p_{T}$ instead of $p$ in (1)(3). In fact, in our previous work [37], the transverse momentum distributions of identified particles $\left(\pi^{ \pm}, \pi^{0}, K^{ \pm}, p, \bar{p}\right.$, and $J / \psi$ ) produced in proton-proton, proton- (deuteron) nucleus, and nucleus-nucleus collisions at the Relativistic Heavy Ion Collider and related energies are studied by using the multisource thermal model. This means that the present 
model can be used in different interacting systems in a wider energy region.

\section{Conflict of Interests}

The authors declare that there is no conflict of interests regarding the publication of this paper.

\section{Acknowledgments}

This work was partly finished at the State University of New York at Stony Brook, USA. One of the authors Fu-Hu Liu thanks Professor Dr. Roy A. Lacey and the members of the Nuclear Chemistry Group of Stony Brook University for their hospitality. The authors acknowledge the supports of the National Natural Science Foundation of China (under Grant no. 10975095 , no. 11005071, and no. 11247250), the China National Fundamental Fund of Personnel Training (under Grant no. J1103210), the Open Research Subject of the Chinese Academy of Sciences Large-Scale Scientific Facility (under Grant no. 2060205), the Shanxi Scholarship Council of China, and the Overseas Training Project for Teachers at Shanxi University.

\section{References}

[1] S. S. Adler, S. Afanasiev, C. Aidala et al., "Saturation of azimuthal anisotropy in $\mathrm{Au}+\mathrm{Au} \sqrt{S_{N N}}=62-200$ collisions at GeV," Physical Review Letters, vol. 94, no. 23, Article ID 232302, 6 pages, 2005.

[2] S. Afanasiev, C. Aidala, N. N. Ajitanand et al., "Elliptic flow for $\sqrt{S_{N N}}=200 \mathrm{GeV}$," Physical Review Letters, vol. 99, no. 5, Article ID 052301, 6 pages, 2007.

[3] R. A. Lacey, "The role of elliptic flow correlations in the discovery of the sQGP at RHIC," Nuclear Physics A, vol. 774, no. 1-4, pp. 199-214, 2006.

[4] G. Gustafson, "String models," Nuclear Physics A, vol. 566, pp. 233-244, 1994.

[5] K. Werner, "Strings, pomerons and the VENUS model of hadronic interactions at ultrarelativistic energies," Physics Reports, vol. 232, no. 2-5, pp. 87-299, 1993.

[6] K. Werner, "Forming quark matter droplets in nuclear collisions," Nuclear Physics A, vol. 566, pp. 477-481, 1994.

[7] H. Sorge, H. Stöcker, and W. Greiner, "Relativistic quantum molecular dynamics approach to nuclear collisions at ultrarelativistic energies," Nuclear Physics A, vol. 498, pp. 567-576, 1989.

[8] H. Sorge, A. von Keitz, R. Mattiello, H. Stöcker, and W. Greiner, "Baryon stopping, flow and equilibration in ultrarelativistic heavy ion collisions," Nuclear Physics A, vol. 525, pp. 95-103, 1991.

[9] A. Jahns, C. Spieles, R. Mattiello et al., "Antibaryon shadowing in massive $\mathrm{Au}+\mathrm{Au}$ collisions at $11 \mathrm{~A} \mathrm{GeV,"} \mathrm{Nuclear} \mathrm{Physics} \mathrm{A,} \mathrm{vol.}$ 566, pp. 483-486, 1994.

[10] X.-N. Wang, "Role of multiple minijets in high-energy hadronic reactions," Physical Review D, vol. 43, no. 1, pp. 104-112, 1991.

[11] X.-N. Wang and M. Gyulassy, "Hijing: a Monte Carlo model for multiple jet production in $p p, p A$, and $A A$ collisions," Physical Review D, vol. 44, no. 11, pp. 3501-3516, 1991.
[12] X.-N. Wang and M. Gyulassy, "Gluon shadowing and jet quenching in $A+A$ collisions at $\sqrt{s}=200 A$ GeV," Physical Review Letters, vol. 68, no. 10, pp. 1480-1483, 1992.

[13] Z.-W. Lin, C. M. Ko, B.-A. Li, B. Zhang, and S. Pal, "Multiphase transport model for relativistic heavy ion collisIons," Physical Review C, vol. 72, no. 6, Article ID 064901, 29 pages, 2005.

[14] K. Tywoniuk, I. C. Arsene, L. Bravina, A. B. Kaidalov, and E. Zabrodin, "Gluon shadowing in the Glauber-Gribov model at HERA," Physics Letters B, vol. 657, no. 4-5, pp. 170-175, 2007.

[15] L. V. Bravina, L. P. Csernai, P. Levai, N. S. Amelin, and D. Strottman, "Fluid dynamics and Quark Gluon string modelwhat we can expect for $\mathrm{Au}+\mathrm{Au}$ collisions at $11.6 \mathrm{AGeV} / \mathrm{c}$," Nuclear Physics A, vol. 566, pp. 461-464, 1994.

[16] D. Kharzeev, E. Levin, and L. McLerran, "Parton saturation and $N_{\text {part }}$ scaling of semi-hard processes in QCD," Physics Letters B, vol. 561, no. 1-2, pp. 93-101, 2003.

[17] K. J. Eskola, K. Kajantie, P. V. Ruuskanen, and K. Tuominen, "Scaling of transverse energies and multiplicities with atomic number and energy in ultrarelativistic nuclear collisions," Nuclear Physics B, vol. 570, no. 1-2, pp. 379-389, 2000.

[18] B.-A. Li and C. M. Ko, "Formation of superdense hadronic matter in high energy heavy-ion collisions," Physical Review C, vol. 52, no. 4, pp. 2037-2063, 1995.

[19] B. Zhang, "ZPC 1.0.1: a parton cascade forultrarelativistic heavy ion collisions," Computer Physics Communications, vol. 109, no. 2-3, pp. 193-206, 1998.

[20] B. Zhang, "Erratum to "ZPC 1.0.1: a parton cascade for ultrarelativistic heavy ion collisions" [Comput. Phys. Commun. 109 (1998) 193-206]," Computer Physics Communications, vol. 111, no. 1-3, p. 276, 1998.

[21] R. B. Clare and D. Strottman, "Relativistic hydrodynamics and heavy ion reactions," Physics Reports, vol. 141, no. 4, pp. 177-280, 1986.

[22] U. Ornik, R. M. Weiner, and G. Wilk, "Hydrodynamical beam jets in high energy hadronic collisions," Nuclear Physics A, vol. 566, pp. 469-472, 1994.

[23] J. Dias de Deus and J. G. Milhano, "A simple evolution equation for rapidity distributions in nucleus-nucleus collisions," Nuclear Physics A, vol. 795, no. 1-4, pp. 98-108, 2007.

[24] J. L. Albacete, "Particle multiplicities in lead-lead collisions at the CERN large hadron collider from nonlinear evolution with running coupling corrections," Physical Review Letters, vol. 99, no. 26, Article ID 262301, 4 pages, 2007.

[25] H. J. Drescher, M. Hladik, S. Ostapchenko, T. Pierog, and K. Werner, "Parton-based Gribov-Regge theory," Physics Reports, vol. 350, no. 2-4, pp. 93-289, 2001.

[26] K. Werner, F.-M. Liu, and T. Pierog, "Parton ladder splitting and the rapidity dependence of transverse momentum spectra in deuteron-gold collisions at the $\mathrm{BN}$ relativistic heavy ion collider," Physical Review C, vol. 74, no. 4, Article ID 044902, 11 pages, 2006.

[27] E. K. G. Sarkisyan and A. S. Sakharov, "Multihadron production features in different reactions," in Multiparticle Dynamics: 35th International Symposium on Multiparticle Dynamics and the Workshop on Particle Correlations and Femtoscopy, vol. 828 of AIP Conference Proceedings, pp. 35-41, Kroměříž, Czech Republic, August 2005.

[28] E. Kokoulina, "The description of $p p$ interactions with very high multiplicity at $70 \mathrm{GeV} / \mathrm{c}$," Acta Physica Polonica B, vol. 35, no. 1, pp. 295-302, 2004. 
[29] D. Kharzeev, Y. V. Kovchegov, and K. Tuchin, "Cronin effect and high- $p_{T}$ suppression in $p A$ collisions," Physical Review D, vol. 68, no. 9, Article ID 094013, 23 pages, 2003.

[30] F.-H. Liu, "Longitudinal and transverse flows of protons in 28A GeV Au-Au collisions," Europhysics Letters, vol. 63, no. 2, pp. 193-199, 2003.

[31] F.-H. Liu, N. N. Abd Allah, D.-H. Zhang, and M.-Y. Duan, "Angular distributions of target black fragments in nucleusnucleus collisions at high energy," International Journal of Modern Physics E, vol. 12, no. 5, pp. 713-723, 2003.

[32] F.-H. Liu, N. N. A. Allah, and B. K. Singh, "Dependence of black fragment azimuthal and projected angular distributions on polar angle in silicon-emulsion collisions at $4.5 \mathrm{~A} \mathrm{GeV} / \mathrm{c}$," Physical Review C, vol. 69, no. 5, Article ID 057601, 4 pages, 2004.

[33] F.-H. Liu, "Unified description of multiplicity distributions of final-state particles produced in collisions at high energies," Nuclear Physics A, vol. 810, no. 1-4, pp. 159-172, 2008.

[34] F.-H. Liu and J.-S. Li, "Isotopic production cross section of fragments in ${ }^{56} \mathrm{Fe}+p$ and ${ }^{136} \mathrm{Xe}\left({ }^{124} \mathrm{Xe}\right)+\mathrm{Pb}$ reactions over an energy range from $300 \mathrm{~A}$ to $1500 \mathrm{~A} \mathrm{MeV,"}$, Physical Review C, vol. 78, no. 4, Article ID 044602, 13 pages, 2008.

[35] M. Apollonio, A. Artamonovf, A. Bagulyal et al., "Forward production of charged pions with incident $\pi^{ \pm}$on nuclear targets measured at the CERN PS," Nuclear Physics A, vol. 821, no. 1-4, pp. 118-192, 2009.

[36] P. Braun-Munzinger, "Hadron production in nuclear collisions and the QCD phase boundary," Talk given at 45 years of nuclear theory at Stony Brook: a tribute to Gerald E. Brown, Stony Brook University, Stony Brook, NY, USA, (will be published as a special issue in Nuclear Physics A), 2013, http://tonic.physics.sunysb.edu/gerrybrown/program2.html.

[37] Y.-Q. Gao, C.-X. Tian, M.-Y. Duan, B.-C. Li, and F.-H. Liu, "Transverse momentum distributions of identified particles produced in $p p, p(d) A$, and $A A$ collisions at high energies," Pramana-Journal of Physics, vol. 79, no. 6, pp. 1407-1423, 2012. 

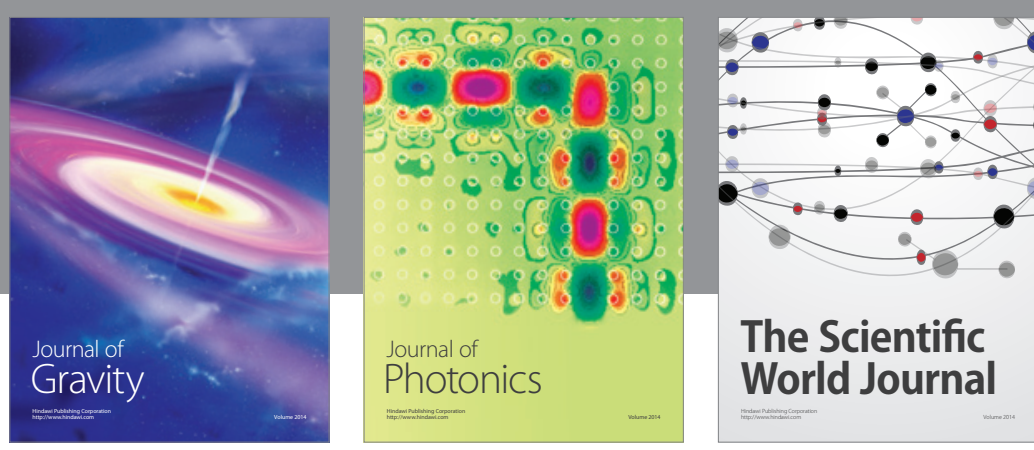

The Scientific World Journal
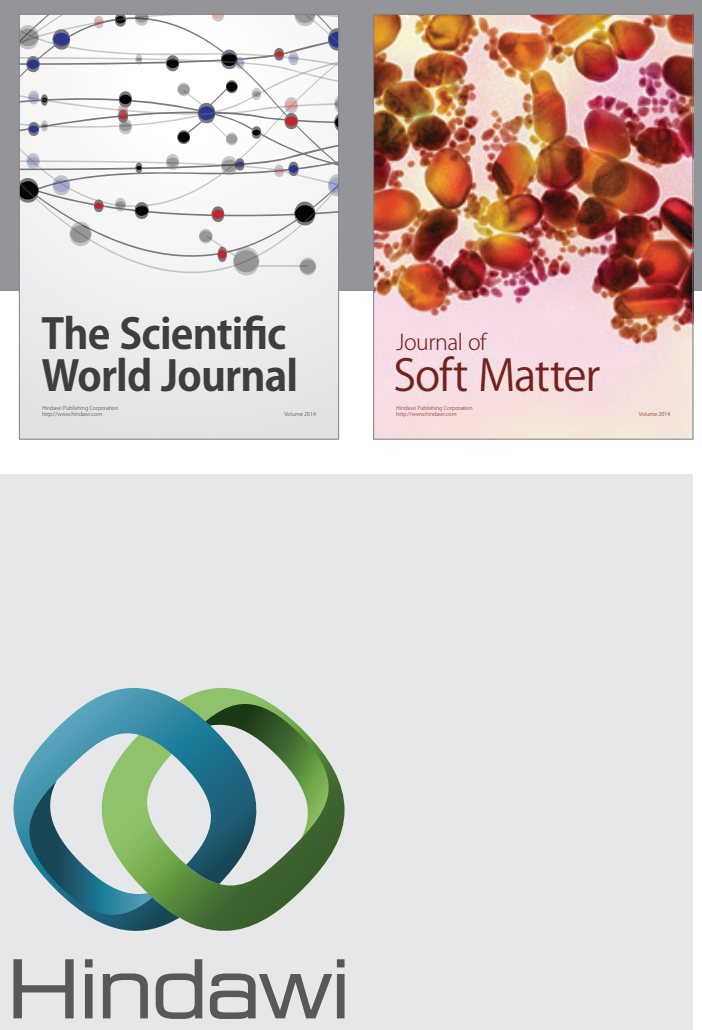

Submit your manuscripts at

http://www.hindawi.com

nternational Journal of

Statistical Mechanics
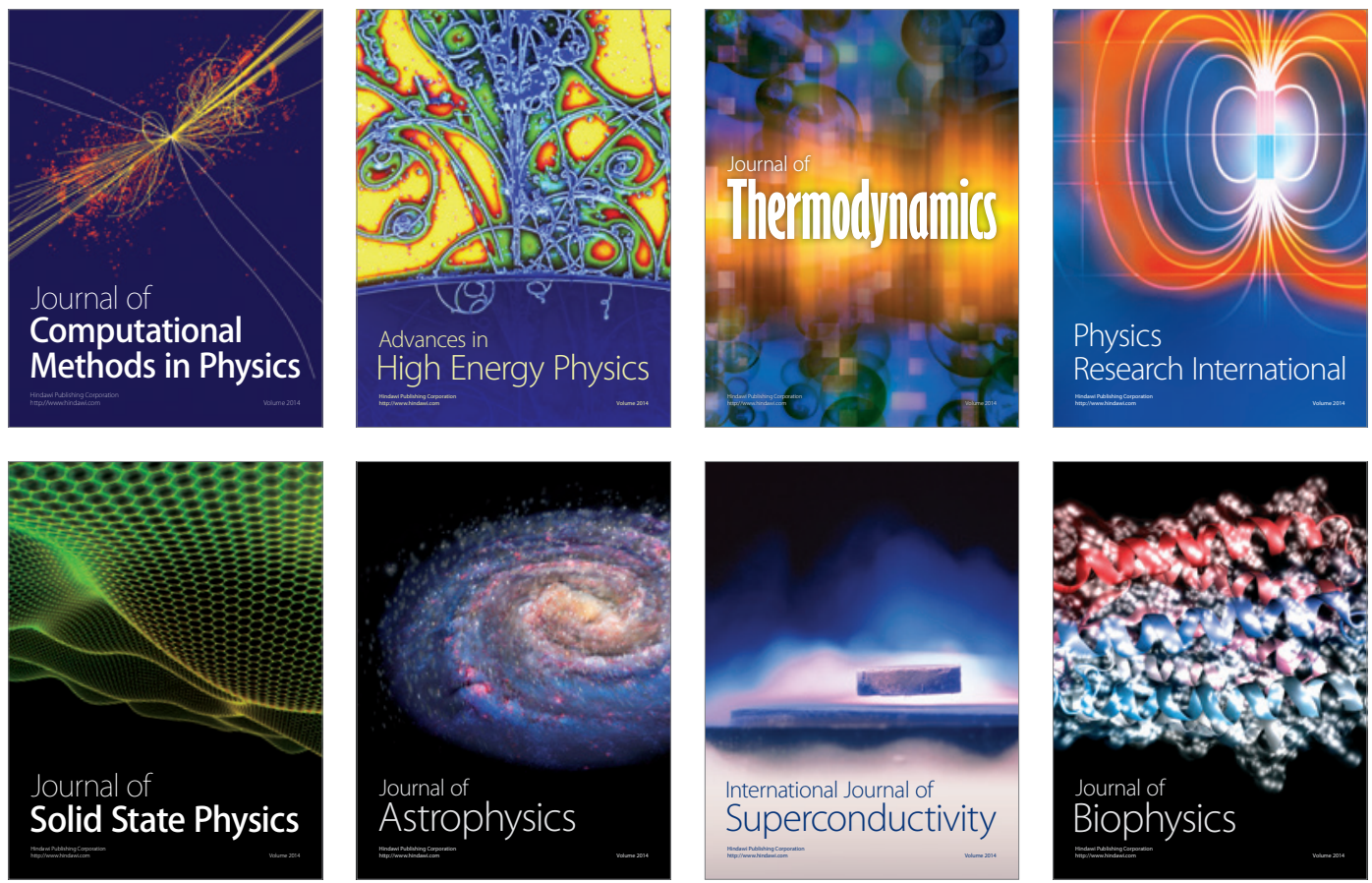
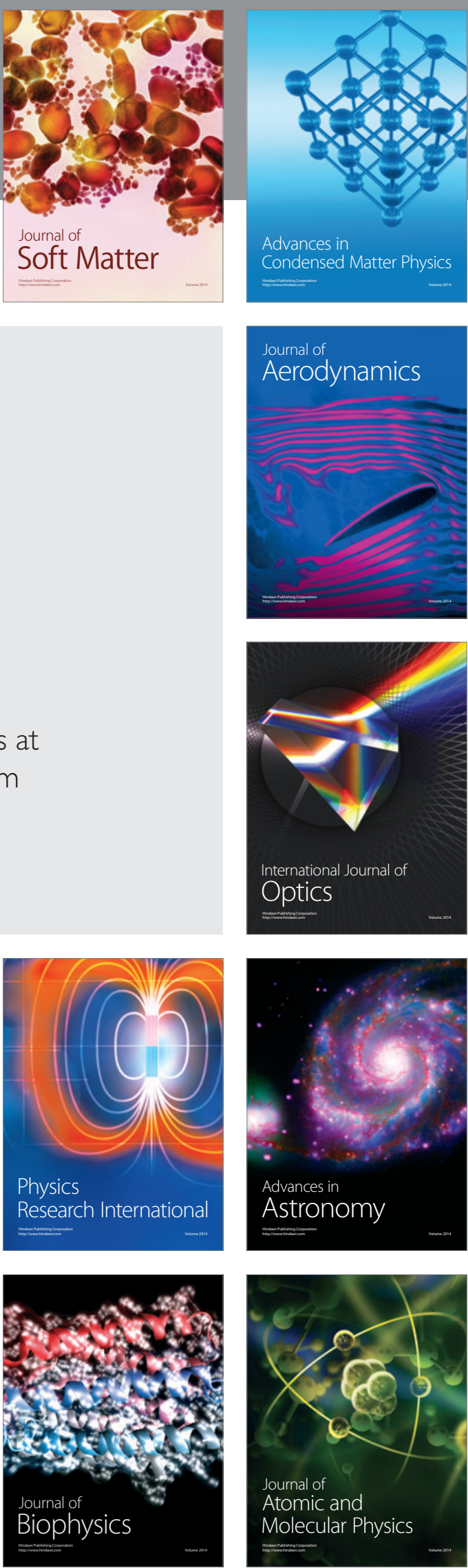